\title{
Speech perception with temporally patterned noise maskers.
}

\author{
Maury Courtland, Louis Goldstein, and Jason D. Zevin
}

December 12, 2019

\begin{abstract}
In the real world, speech perception frequently occurs under adverse listening conditions. Laboratory studies have identified distinct phenomena associated with more or less constant sources of noise (energetic masking), and competing verbal information (informational masking). One issue complicating direct comparisons between them is that common paradigms for studying energetic and informational masking differ along many dimensions, in particular, informational masking is almost always measured using linguistically meaningful information. We have developed a paradigm that uses temporally patterned noise, with the goal of comparing energetic and informational masking under more comparable conditions. We hypothesized that listeners would be able to take advantage of the structure in the masking noise, providing a processing advantage over energetic masking. The initial experiment provides strong evidence for this hypothesis, but conceptual replications did not produce the same pattern of results - at least with respect to measures of central tendency. A direct replication of the first experiment did not replicate the large differences in the means but a final experiment strengthening the effect did. Interestingly, however, exploratory analyses across all five experiments reveal robust evidence that patterned noise conditions produce increased individual variability. Further, we observed strong correlations, specifically between the patterned conditions. We attribute these findings to as yet unidentified cognitive ability differences allowing some participants to benefit from the use of additional temporal information while others are hurt by the addition of unusable distracting information. Hypothesized predictive measures of task performance, such as working memory, inhibitory control, and musical experience did not correlate with performance, however.
\end{abstract}

\section{Introduction}

Speech perception must regularly overcome the noisy nature of the real-world contexts in which it occurs. Previous studies have delineated two distinct sources causing processing difficulty: interfering background noise, i.e., energetic masking, and distracting signals that are meaningful but irrelevant, i.e., informational masking (Pollack 1975, for review see Kidd et al. 2008). They differ, for example, in that the degree of interference from energetic masking is highly correlated with the level of background noise, whereas informational masking is only weakly related to the relative level of the competing signal (e.g. Brungart 2001). When maskers are quiet relative to targets, informational masking is more difficult, as the additional information is distracting. However, at higher levels, the ability to treat the competing signal and target as distinct auditory objects enhances the ability to specifically attend to the target, making informational masking easier. Energetic and informational masking also seem to engage separate networks in neuroimaging studies (e.g. Scott et al. 2004).

One issue with characterizing the distinction between energetic and informational masking is that the typical paradigms for studying the two differ on many dimensions, preventing direct comparisons between them. Energetic masking is generally created with white or speech-shaped noise, whereas informational masking typically involves one or more additional talkers as an interfering mask (Mattys et al., 2012). As a result, any inferences we draw about informational masking and its difference from energetic masking are constrained to cases in which the competing information is also a linguistic signal. Thus, Scott et al. (2004) attribute their findings of increased superior temporal gyrus activity during informational masking to the need to process more speech. Because informational masking stimuli are always linguistic, it is unclear what the relative contributions in overcoming informational masking are of language-specific processing i.e., filtering unwanted speech by content, Treisman (1964), or general auditory scene 
analysis i.e. segregating masker and target streams using object-based attentional mechanisms, which would also be engaged to selectively attend to speech in an array of non-speech auditory objects, Bregman (1990).

To address this question, we created a patterned, non-linguistic stimulus to isolate the role of non-linguistic attentional mechanisms in informational masking. To allow direct comparison to energetic maskers, we construct maskers using speech-shaped noise correlated with the long-term average spectrum of our target words, and then produce temporal structure by inserting regularly patterned, non-isochronous, silences into the masker (see Sheft and Yost 2008 for a discussion of information v. structure). The complexity of the masker compared to previous isochronous studies (e.g. Miller and Licklider 1950) aims to recruit the same mechanisms that process the complex temporal regularities of an interfering speech signal in canonical informational masking. The regularity of the masker, on the other hand, differs from previous complex maskers (e.g. Festen and Plomp 1990) and provides the temporal structure present in stimuli designed to induce auditory stream segregation (Bregman, 1990). In contrast to previous studies, our maskers also precisely control for both global and local SNR, ensuring that performance differences arise solely from the presence or absence of information in the maskers.

We hypothesized that listeners would be able to take advantage of the regular temporal structure of the masking noise, providing a processing advantage over energetic masking. This was supported by work such as Andreou et al. (2011), who found that tone-on-tone maskers' temporal regularity facilitates target perception when tones repeat every $100-250 \mathrm{~ms}$ and spectral separation alone is insufficient to segregate streams. The parameters of our maskers adhere to these criteria for facilitating target perception: the maskers are interleaved with silences in $125 \mathrm{~ms}$ windows, and their spectral profiles match the long-term average of the targets they mask. We predicted that speech perception would analogously improve when our maskers contained temporal structure.

An initial experiment provided strong evidence for this hypothesis (Lander-Portnoy, 2016), but conceptual replications and a direct replication reported here (Experiments 2-4) did not produce significant differences in mean accuracy. A final experiment (Experiment 5) using a hypothesized method for strengthening the effect did replicate the original effect, however. In this paper, we examine measures of variability and patterns of correlation across conditions in both the initial and follow-up experiments. Interestingly, we find that these measures are very similar across experiments, suggesting that some as yet unidentified difference among participants is responsible for the systematic variability in performance we observe across conditions. We then discuss the robustness of our findings across experiments and identify mechanisms implicated in this systematic variability.

\section{Experiment 1}

In this experiment, we asked participants to identify words presented with maskers comprising patterns of speechshaped noise interrupted by short silent intervals. The duration and timing of the silent intervals were irregular, and in a subset of the trials, was repeated twice before the presentation of the masked speech stimulus. We predicted that participants would be able to make use of the repeating pattern in the stimulus to improve their performance on the word recognition task.

\section{$2.1 \quad$ Methods}

\subsubsection{Participants}

Sixty undergraduate students at the University of Southern California participated in exchange for course credit. No personally identifiable information was gathered about participants. All participants had self-reported normal hearing and were monolingual speakers of English. None had previously participated in speech perception experiments in the lab. 


\subsubsection{Stimuli}

The 108 target items were polysyllabic words selected from The Nationwide Speech Project Corpus (Clopper and Pisoni, 2006). Speakers were selected at random to include 9 speakers (4-5 female, 4-5 male) from each of the 6 United States dialect regions in the corpus. The corpus consists of white 18-25 year old native speakers of English with no history of hearing or speech disorders.

Target items were manually extracted from the corpus using Praat (Boersma and Weenink, 2012) to allow precise control over onset timing of the target word. A three second sound file was created with the target beginning at the $2 \mathrm{~s}$ mark. This file was then added to a $3 \mathrm{~s}$ sound file containing the masker, created as described below. Stimuli were masked by speech shaped noise (SSN) correlated to the long term average spectrum (LTAS) of the aggregate of all targets. A $100^{\text {th }}$ order linear predictor coefficient (LPC) filter was calculated from the concatenated targets. For each trial, 3 second white noise samples were generated and filtered using this $100^{\text {th }}$ order LPC filter to match its spectral profile to the LTAS. It should be noted that this spectrum differs somewhat from an individual target's LTAS. While matching SSN to target LTAS increases masker fit, it undesirably provides participants anticipatory spectral information about the upcoming target. After filtering, maskers' volumes were set at a signal-to-noise ratio (SNR) of $-10 \mathrm{~dB}$ relative to their targets.

To create regular temporal information, we divide the noise into $8 \mathrm{~Hz}$ windows (125ms each). In each window, we silence a portion of the previously constant masker, creating periods in which to glimpse the target unobstructed (see Cooke 2003 for Glimpsing). We then create 4 window types varying in the silence's duration and location within the window, as shown in Figure 1. The first of these window types contained 25ms (20\%) silence at the beginning of the window, with the remainder SSN noise. Type 2 contained 100ms (80\%) silence at the beginning. Type 3 and 4 contained $25 \mathrm{~ms}$ and $100 \mathrm{~ms}$ of silence respectively in the middle of the windows. We then randomly permute two of each window type and concatenate them to create a 1 second rhythmic pattern (for example, see Fig. 1, right). Given that each pattern's information lies wholly in the ordering of windows and not in their composition or selection, the amount of noise power, global SNR, and total noise duration remain constant across patterns.
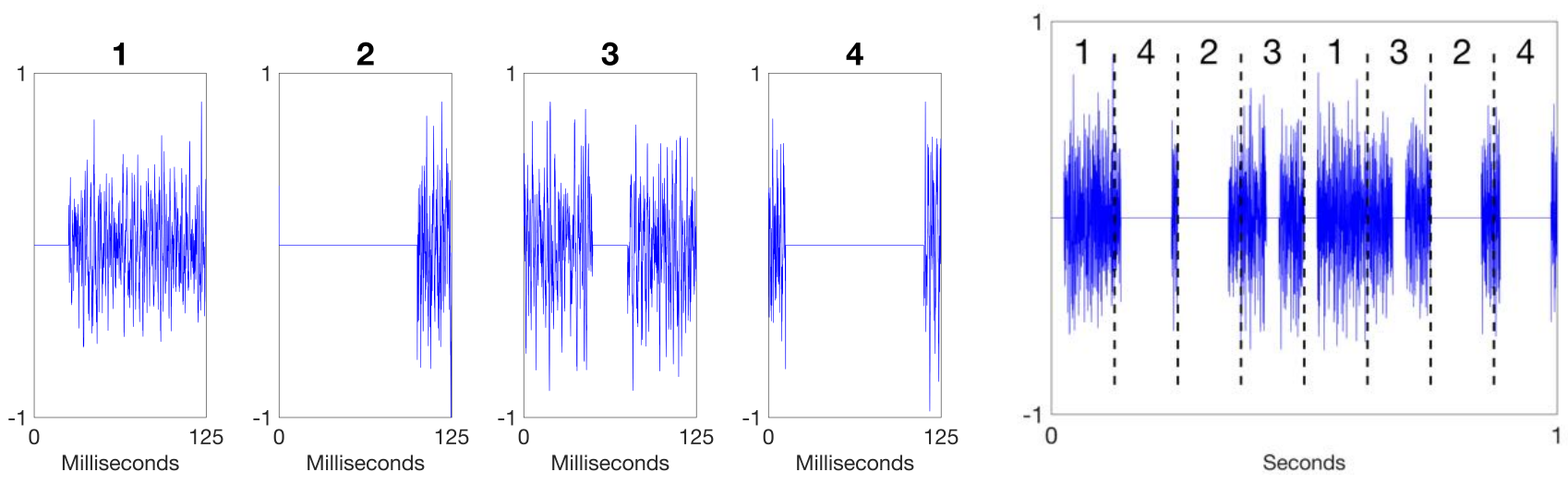

Figure 1: We utilize four different types of $125 \mathrm{~ms}$ windows (left) to construct noise patterns (right). Windows 1 and 3 contain $20 \%$ silence $(25 \mathrm{~ms})$ and $80 \%$ noise (100ms), while windows 2 and 4 contain $80 \%$ silence $(100 \mathrm{~ms})$ and $20 \%$ noise (25ms). Additionally, in windows 1 and 2, the beginning of the silence is aligned with the beginning of the window, whereas in windows 3 and 4 , the middle of the silence is aligned with the middle of the window. We then create a pattern by randomly permuting two of each window type (denoted at top). As windows are $125 \mathrm{~ms}$ long, and each pattern contains 8 windows, patterns are 1 second long.

For each target, we create three conditions to occur in different lists: one that affords masking pattern prediction, one that does not but provides the same glimpses (i.e. local SNR) and forward and backward masking effects (Widin and Viemeister, 1979; Smiarowski and Carhart, 1975), and one with unrelated random patterns, as shown in Figure 2. The first condition repeats the masking pattern twice in the preamble allowing participants to recognize it prior to the onset of the masked target in the third second. The second condition utilizes the same masking pattern as the first condition to control for glimpses it affords, but contains two random preamble patterns providing no anticipatory information. The third condition includes three different patterns that serve as a task performance baseline. The two random preamble patterns in condition two and all patterns in condition three only occur once per experiment and are thus novel when participants hear them. In contrast, the noise pattern that serves as masker in conditions one and two, which we call "Pattern A", is repeated every trial of condition one or two. 
Because condition one comprises a threefold repetition of Pattern A, we call this condition AAA. Because condition two contains two random patterns followed by Pattern A, we call this condition XYA. Lastly, because condition three contains three random patterns, we call this condition XYZ. Given that AAA contains all temporal information, XYA just cross-trial information, and XYZ no information, we expect participants' performance in these conditions to correlate with the amount of available temporal information (i.e. $A A A>X Y A>X Y Z$ ).
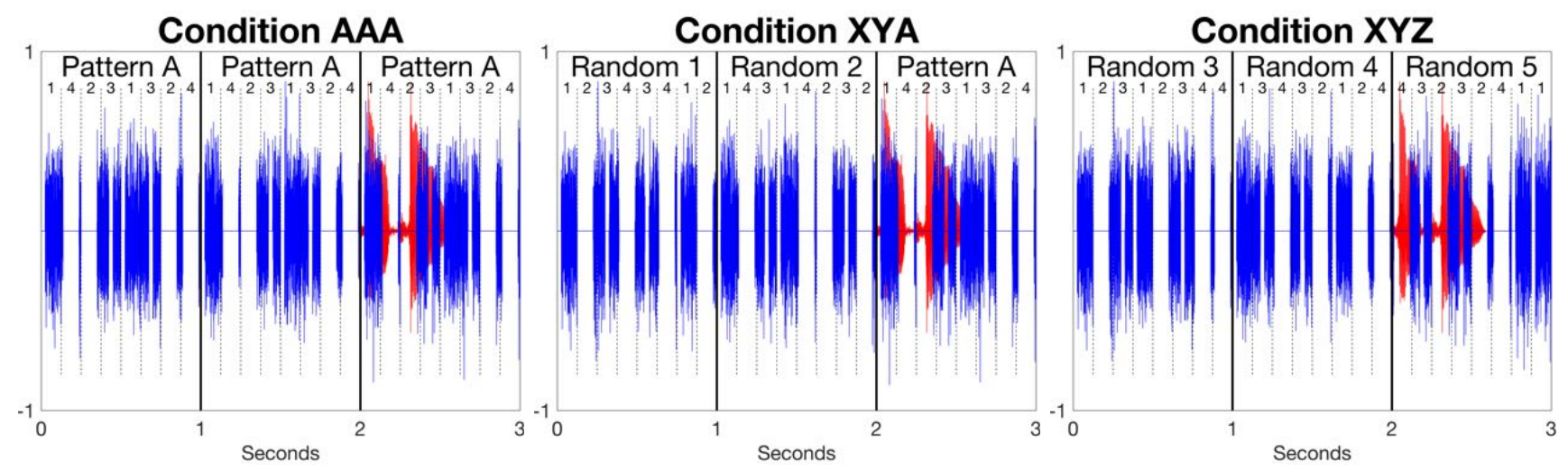

Figure 2: Wave forms showing the three experimental conditions with SSN in blue and target word in red. AAA (left) affords masking pattern information twice before target presentation. XYA (center) affords the same glimpsing windows as AAA, but no information about the upcoming masking pattern. XYZ (right) provides the same global SNR as AAA \& XYA but contains no temporal regularity.

\subsubsection{Procedure}

We divided the 108 targets equally into each condition (36 each) and grouped trials into 4 blocks (27 each). Each block contained 9 triplets of one trial per condition permuted randomly to provide uniform exposure to each condition. This resulted in 12 lists ( $3 \times 4$ Latin square, 5 participants per) counterbalancing block and condition.

Testing occurred in a noise attenuating booth. Stimuli were presented over headphones in mono at a sampling rate of 44,100 Hz at 16 bits. Volume was set at a comfortable level consistent across participants. Participants typed their responses in a free response text box and advanced to the next trial when ready. Responses were automatically checked for correctness and those marked incorrect were checked by hand for misspellings. No partial credit was given. Initial separate scoring for inflectional errors and lemma identification proved uninformative, and are not presented here.

\subsubsection{Ancillary cognitive measures}

Participants were also tested in measures of working memory and inhibitory control. The working memory task was an ordered digit span recall task in which digits (0-9) were presented sequentially on screen for $250 \mathrm{~ms}$ each, with a $750 \mathrm{~ms}$ pause after each digit. Digits were sampled uniformly at random with replacement and a correct response consisted of the digits ranked in ascending order. Trials gradually increased in length from three digits to eight digits with five trials occurring per span length.

Performance on the Digit Span task was calculated as a simple percentage of total trials correct. No partial credit was given.

The inhibitory control task presented a square that was red or yellow on the left or right side of the screen. Participants clicked the left or right mouse button according to color and not screen position. Incongruent trials are those when correct mouse response and screen position are opposite.

Performance on the inhibitory control task is measured by the difference between the mean response time of correct congruent trials and correct incongruent trials. We exclude responses less than $200 \mathrm{~ms}$ or more than $1500 \mathrm{~ms}$ due to their probable cause by inadvertent click and distraction, respectively. We then exclude outliers further than $3 \sigma$ away. 


\subsection{Results}

\subsubsection{Hypothesis Testing}

Distributions and means for each condition are shown in Figure 3. To test for differences between conditions, we use a multilevel mixed-effects logistic regression model with accuracy as the dependent variable, condition as a fixed effect, and participant and item as random effects modeled as random intercepts (implemented in Stata 13.1).

The model reveals a significant main effect of condition, $\chi^{2}(2, N=60)=16.41, p<.0005$. Pairwise comparison between conditions indicate significant differences between AAA and XYA, $\beta=-.25, z=-3.36, p=.001$ as well as AAA and XYZ, $\beta=-.27, z=-3.67, p<.001$ but no significant difference between XYA and XYZ, $\beta=-.02, z=-0.30, p=.76$.
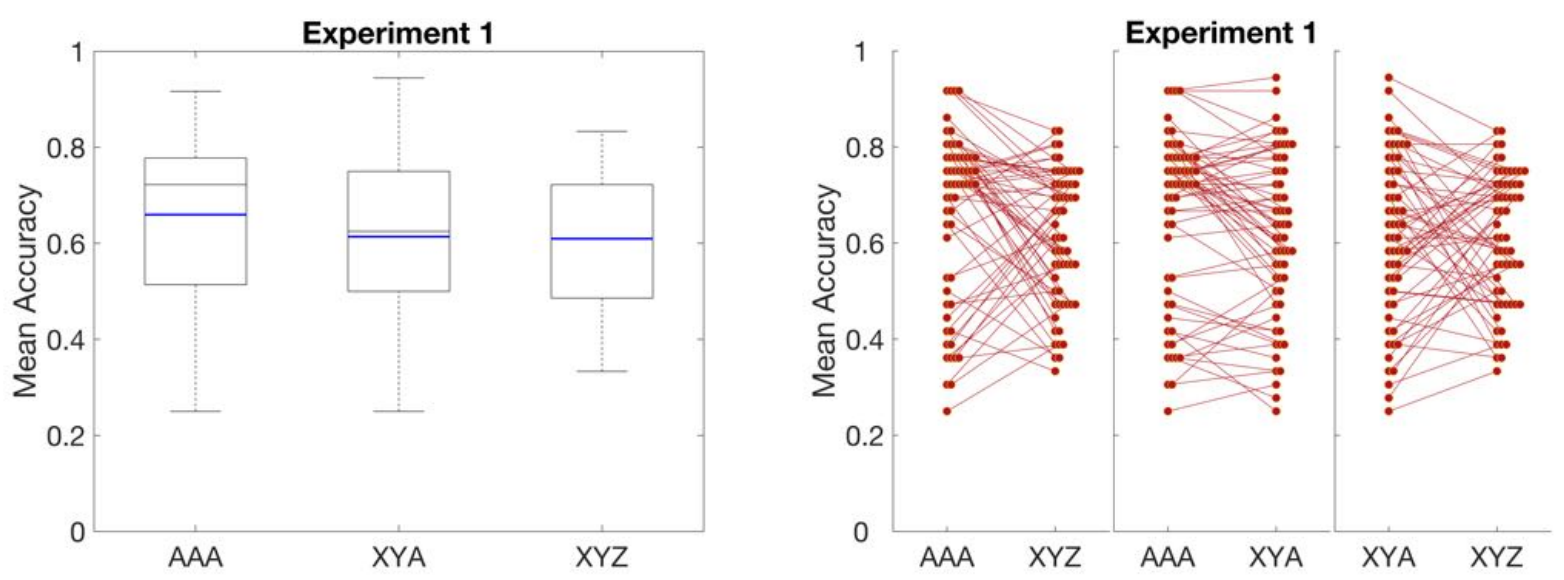

Figure 3: Results of the word recognition task performance by condition. Significant differences in sample means (blue, left) are found between AAA and XYZ as well as AAA and XYA, but not XYA and XYZ. Performance distributions (right) are represented as vertical histograms (i.e. histograms rotated clockwise $90^{\circ}$ ) with each circle representing a participant. These distributions show significant variance differences between AAA and XYZ, as well as XYA and XYZ, but not AAA and XYA. Lines connect participants' performances in each condition and visually represent correlations between conditions.

\subsubsection{Exploratory Analyses}

Following the unexpected results of several follow-up experiments, we reanalyzed the results of previous experiments to help explain our findings. These analyses are presented in the "Exploratory Analyses" sections throughout this paper.

Variance Differences As shown in Figure 3, large differences in variance were observed among the conditions. We tested differences in variance using Bartlett's Test (Bartlett, 1937), and found a significant difference between the variances of AAA, $s=.18$, and XYZ, $s=.14, T(1)=3.99, p<.05$, as well as a marginally significant difference between XYA, $s=.17$, and XYZ, $s=.14, T(1)=2.65, p=.10$. No difference was observed between AAA, $s=.18$, and XYA $s=.17, T(1)=.14, p=.71$.

Correlations between conditions As shown in Figure 4, performance is correlated across conditions. There is a strong correlation between performance in the AAA condition and performance in the XYA condition, $r(58)=$ $0.79, p<.0001$, whereas the correlation between AAA and XYZ, $r(58)=0.32, p<.01$, and XYA and XYZ, $r(58)=0.26, p<.05$, are moderate at best.

Using a Fisher z-transformation (Fisher, 1915; Lowry, 2001), we compare the correlations between conditions found above. We find AAA/XYA to be a significantly stronger correlation than AAA/XYZ, $z=3.95, p<.0001$, and 

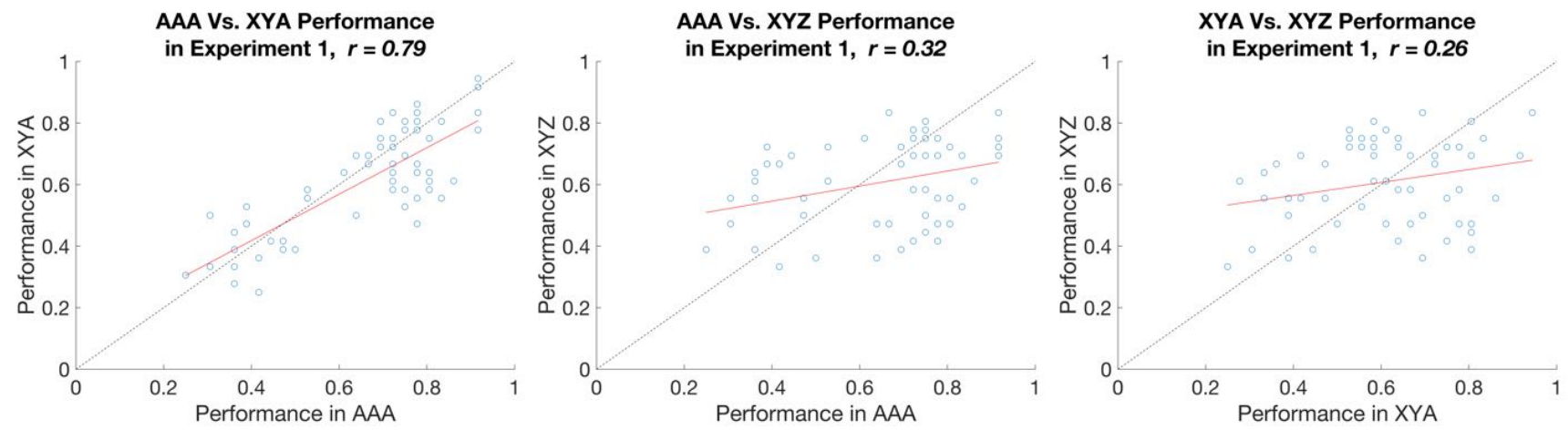

Figure 4: We find a strong correlation in Experiment 1 between the conditions containing long-term information but not between either of these and baseline. A least mean squares linear regression line is drawn on the plots in red and the identity line in black.

also significantly stronger than XYA $/ \mathrm{XYZ}, z=4.30, p<.0001$. We do not find a significant difference in strength between $\mathrm{AAA} / \mathrm{XYZ}$ and XYA/XYZ, $z=.35, p=.36$.

Finally, we perform hierarchical regression to test whether XYA explains additional variance in AAA performance beyond baseline (XYZ). We find that the model using both XYA and XYZ as predictors explains significantly more variance in AAA than the model using XYZ alone, $F(1,57)=85.89, p<.0001$. While the model using XYZ alone explains a significant amount of AAA variance, $F(1,58)=6.54, p=.01$, the addition of XYZ as a predictor does not explain significantly more variance than a model with XYA as the sole predictor, $F(1,57)=2.15, p=.15$. XYA also explains a significant amount of AAA variance alone, $F(1,58)=97.91, p<.0001$.

\subsubsection{Cognitive Ability Measures}

Inhibitory Control We observe a mean incongruence effect of $29 \mathrm{~ms}(95 \% \mathrm{CI}=(22 \mathrm{~ms}, 37 \mathrm{~ms}))$. A t-test reveals this mean difference effect to be significant, $t(58)=7.54, p<.0001$. Performance in the inhibitory task is not significantly correlated with overall performance in the word recognition task, $r(58)=-.21, p=.11$, nor with the benefit from repeated masking patterns (i.e. the difference between mean scores in the AAA and XYZ conditions), $r(58)=-.09, p=.52$.

Digit Span Participants' mean score for digit span recall was $76 \%,(95 \%$ CI $=(72 \%, 79 \%))$. We observe no significant correlation of digit span with overall word recognition performance, $r(58)=.11, p=.39$, nor with a difference score computed to indicate the benefit of repeating the masking pattern (condition $A A A-X Y Z$ ), $r(58)=.04, p=.76$. Similarly, no evidence was observed for a relationship between inhibitory control and working memory capacity, $r(58)=-.05, p=.70$.

\subsection{Discussion}

Participants in this experiment were more accurate on trials when the temporal pattern was repeated (AAA) than in two control conditions. To foreshadow the results of the following studies, this difference in means was not reproduced in either conceptual or direct replications. Exploratory analyses, however, revealed that trials on which the stimuli were masked by the pattern repeated throughout the experiment (AAA and XYA) both produced increased individual variability, and further, that performance in these conditions was strongly correlated, explaining a significant amount of performance variability over baseline. Some participants improved relative to the baseline (XYZ) condition, whereas other participants performed more poorly when the masker was repeated. We collected working memory and inhibitory control measures, which have been shown to correlate with speech perception in noise in prior research (for review see Dryden et al. 2017; Akeroyd 2008), but these did not correlate with overall performance, nor with the repetition advantage. Nonetheless, the results suggest that some stable difference or differences among participants makes the repetition of the noise pattern beneficial for some and detrimental to others. 


\section{Experiment 2}

Based on the results of Experiment 1, we hypothesized two possible mechanisms responsible for the significant performance difference between the repeated condition (AAA) and the control condition (XYA). The first is a global perceptual grouping mechanism akin to stream segregation (Bregman, 1990) by which the noise pattern is perceived as distinct from the target speech. If the target speech is perceived as a separate object, attending to it will increase its perceptibility, and thus task performance. The alternative hypothesis is a local computational mechanism akin to beat induction and matching (Honing, 2012; Winkler et al., 2009) which calculates intervals between glimpsing windows so that attention can be allocated to the unmasked portions of the speech signal (Cooke, 2003). This experiment was devised to distinguish between these two hypotheses by interrupting streaming processes using short silences.

Auditory object formation is an online process that requires signal continuity and attention to construct a representation of a sound (Snyder et al., 2006; Shamma et al., 2011). When attention shifts away from the sound or the signal is interrupted by silence, the process rapidly resets and must begin anew (Cusack et al., 2004). We use this rapid resetting after interruption to defeat perceptual grouping by inserting silences between noise patterns. Because the silences are regular, the temporal predictability of glimpses remains unaffected. This selective affordance for local computation allows us to probe which phenomenon underlies the advantage observed in Experiment 1. If perceptual grouping is at play, we expect a null result. If local computation is at play, we expect to replicate Experiment 1.

\subsection{Methods}

\section{Participants and Procedure}

60 new participants were recruited from the same population as Experiment 1 in the same manner. The procedure is identical as well except for the removal of the inhibitory control and digit span tasks.

\section{Stimuli}

We generated stimuli in the same manner as Experiment 1 with identical window types, noise spectrum, and signalto-noise ratio. In this experiment, however, we insert $433 \mathrm{~ms}$ silences between noise patterns causing trials to last 3.866s rather than 3.0s. We refer to the conditions in the same manner as Experiment 1 but use the underscores to denote silences: A_A_A, X_Y_A, and X_Y_Z.

\subsection{Results}

\subsubsection{Hypothesis Testing}

We use the same mixed-effects logistic regression model with subjects and words as random intercepts as in Experiment 1. The model reveals no significant main effect of condition, $\chi^{2}(2, N=60)=3.08, p=.21$.

\subsubsection{Exploratory Analyses}

Variance Differences Bartlett's test reveals a significant variance difference between A_A_A $(s=.16)$ and X_Y_Z $(s=.11), T(1)=6.56, p=.01$ and between X_Y_A $(s=.16)$ and X_Y_Z, $T(1)=6.24, p=.01$. No difference in variance was observed between A_A_A and X_Y_A, $T(1)<.01, p=.95$. Thus, as in Experiment 1, variance was greatest when the masking pattern was repeated throughout the experiment, whether it was repeated in the course of a single trial or not, as shown in Figure 5. 

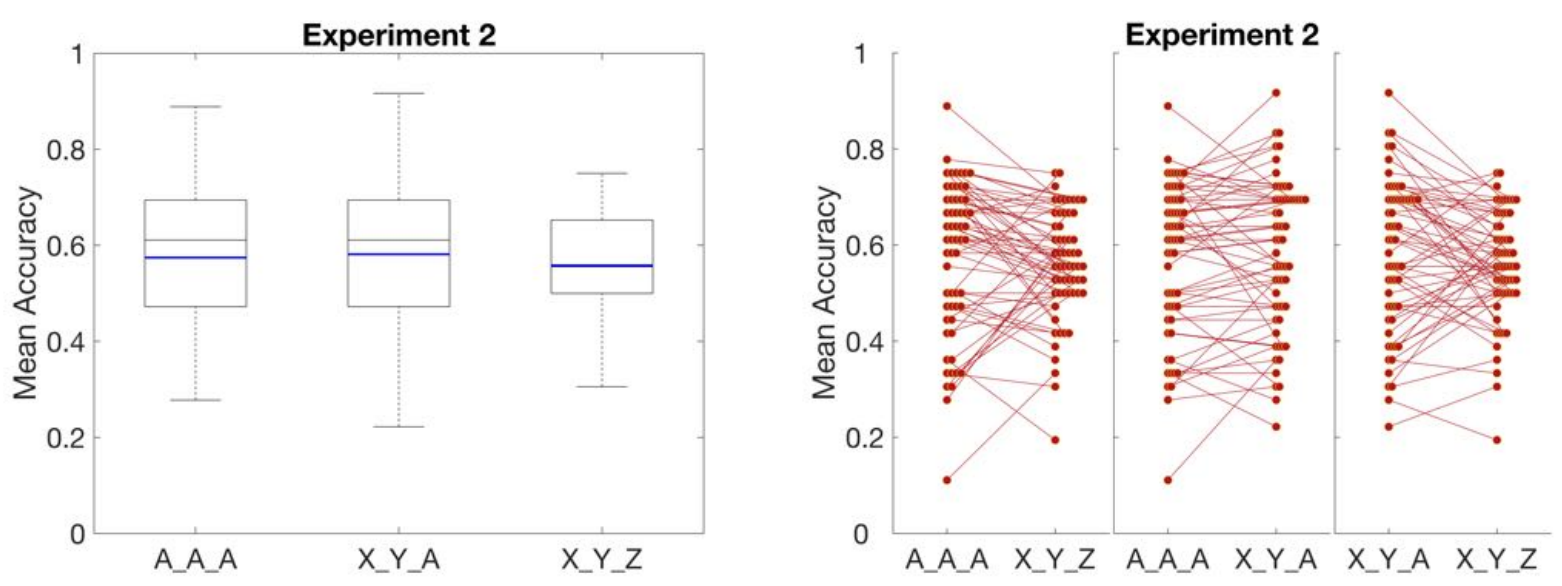

Figure 5: Results of word recognition task with inserted silences (denoted by "_"). We find no significant difference in sample means (blue, left) between A_A_A and either X_Y_A or X_Y_Z, but find a marginally significant difference between X_Y_A and X_Y_Z. Distribution variances are again significantly different between $A_{-}$A_A and $X_{-}$Y_Z, as well as X_Y_A and X_Y_Z, but not A_A_A and X_Y_A. Lines (right) again connect a participant's performances and represent correlation between conditions.

Condition performance correlations We observe a strong correlation between A_A_A and X_Y_A, $r(58)=$ $.79, p<.0001$, as shown in Figure 6 . Moderate correlations were observed between A_A_A and X_Y_Z,$r(58)=$ $.51, p<.0001$ as well as between $\mathrm{X}_{-} \mathrm{Y}_{-} \mathrm{A}$ and $\mathrm{X}_{-} \mathrm{Y} \_\mathrm{Z}, r(58)=.53, p<.0001$.
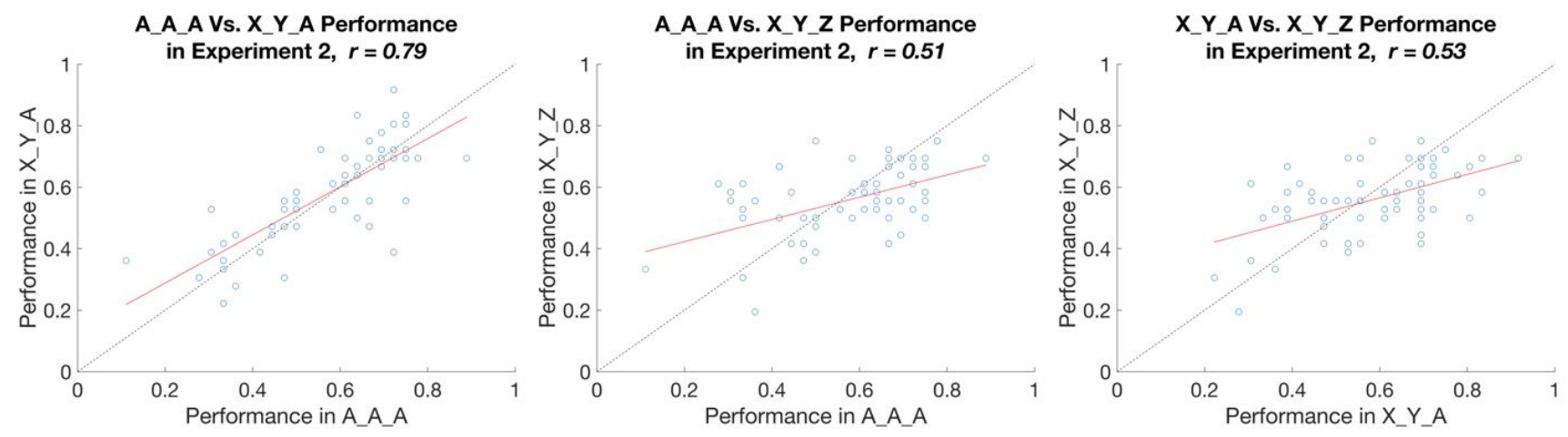

Figure 6: We again find a strong correlation between performances in the long-term information conditions (A_A_A and X_Y_A) in Experiment 2. Interestingly, correlation of both A_A_A and X_Y_A with $X_{-} Y \_Z$ increases relative to Experiment 1. Despite this, the correlation of long-term conditions with each other is still significantly stronger than either with baseline. A LMS regression line is drawn in red and the identity line is drawn in black for reference.

We again test for significant differences in correlation strengths using Fisher's z-transformation. We find A_A_A/X_Y_A to be a significantly stronger correlation than A_A_A/X_Y_Z, $z=2.74, p<.005$, and than X_Y_A/X_Y_Z, $z=2.58, p=.005$. We do not find evidence of a significant correlation strength difference between A_A_A/X_Y_Z

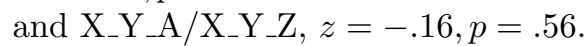

Finally, we again perform hierarchical regression to test whether X_Y_A explains additional A_A_A variance beyond baseline alone. Using both $X_{-} Y_{-} A$ and $X_{-} Y_{-} Z$ as predictors explains significantly more variance than using X_Y_Z alone, $F(1,57)=59.13, p<.0001$. Using $X_{-} \_$Y_Z alone again explains a significant amount of variance, $F(1,58)=20.08, p<.0001$, but adding $\mathrm{X}_{-} \mathrm{Y}_{-} \mathrm{Z}$ does not explain significantly more variance than a model with X_Y_A as the sole predictor, $F(1,57)=1.74, p=.19$. X_Y_A alone again explains significant A_A_A variance, $F(1,58)=96.37, p<.0001$.

\subsection{Experiment 2 Discussion}

We did not find differences in means across conditions. These data must be interpreted in the context of the failure to reproduce the effect of Experiment 1 in replications (Experiments 3 and 4, below), however. Thus, we cannot ascribe 
the lack of an advantage in the AAA condition to a disruption of streaming by the addition of silences. In contrast, we did replicate the pattern of change in variances in both the AAA and XYA conditions. We also replicated the strong correlation between performance in these conditions, further supported by the hierarchical regression findings. This is further evidence that whatever mechanism is driving the individual differences in performance does not depend on streaming, and instead may be a result of long-term memory for the repeated " $\mathrm{A}$ " pattern.

\section{Experiment 3}

This experiment was designed based on results of the tests of a priori hypotheses from Experiments 1 and 2, and without the benefit of the exploratory analyses. We reasoned that the lack of an advantage for the AAA condition in Experiment 2 could be due to interruption of streaming, and sought to test whether object formation could be driven at the single trial level by repeating a masker that the participant has never encountered before.

Attending to objects and encoding them into memory allows participants to identify the object in future trials, thus facilitating task performance. This object persistence in memory with subsequent recall is attested visually in Hollingworth (2005)'s change detection task, where participants performed equivalently whether the object identification was online, in the next trial, or at the end of the experiment. This memory encoding represents features of the object and in relation to the object's context (Sun and Gordon, 2010). Encoding and retrieval of object representations in memory, and their strengthening with exposure, is strongly implicated in our experiments. Given our previous inconclusive results, we perform an experiment to delineate the role of isolated long-term information in affording the initially observed advantage. If previous exposure to the object in question (pattern A) is necessary to segregate streams to defeat masking, then we expect higher performance only in the condition containing long-term information. If, however, the mechanism relies solely on repetitive structure, both conditions containing short-term information should be distinct from baseline, but not each other.

\subsection{Methods}

\section{Participants and Procedure}

60 new participants were recruited from the same population and in the same manner as previous experiments. The word recognition in noise task proceeded the same as in previous experiments. Given the need to recognize and encode regular temporal structure in our experiments, we hypothesize that musical experience may be a good predictor of performance. We therefore include surveys of musical experience that cover musical training and performance, attitudes toward music, and music consumption behavior.

\subsubsection{Stimuli}

Maskers for this experiment were synthesized in the same way as previous experiments, with identical parameters, by inserting silences into long-term average spectrum speech shaped noise to create rhythmic patterns, as in Experiment 1. In this experiment, we include conditions AAA, containing both short-term and long-term temporal information, and XYZ, a baseline containing neither, constructed as in Experiment 1. To test the isolated benefit of short-term temporal information, we also include a condition exhibiting only the repetition of noise patterns within a trial but not their reuse across trials. This condition contains threefold repetition of a novel pattern each trial which we denote with the variable $\mathrm{X}$, giving us condition "XXX".

\subsection{Results}

\subsubsection{Hypothesis Testing}

The same model as Experiments 1 and 2 reveals no significant main effect of condition, $\chi^{2}(2, N=60)=2.07, p=.36$. 

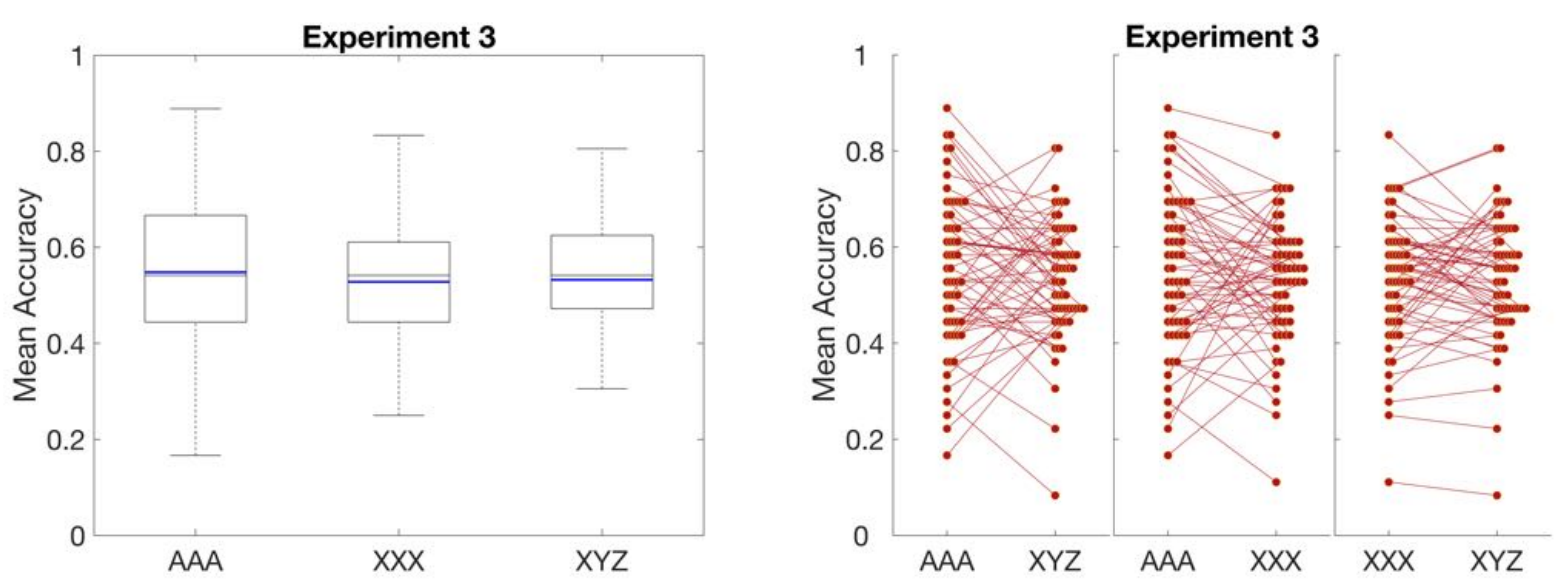

Figure 7: Neither the novel isolated short-term information condition, XXX, nor the previously significant short-term and long-term condition, AAA, differ significantly in their sample means (blue, left) from baseline, XYZ, or from each other. The distribution variance of AAA differs significantly from both XXX and XYZ but the two do not differ from each other. Lines (right) connect a participant's performances and represent correlation between conditions.

\subsubsection{Exploratory Analyses}

Variance Differences Bartlett's test reveals a marginally significant difference between AAA, $s=.16$, and XYZ, $s=.13, T(1)=3.02, p=.08$, and a marginally significant difference between AAA and XXX $s=.13, T(1)=$ $2.69, p=.10$. No difference was observed between XXX and XYZ, $T(1)<.01, p=.92$.

Condition performance correlations We found weak to moderate correlations between performance in the $\mathrm{AAA}$ and XXX conditions, $r(58)=.38, p=.001$, and between AAA and XYZ, $r(58)=.29, p=.01$. A somewhat stronger correlation was found between XXX and XYZ, $r(58)=.56, p<.0001$, perhaps suggesting that variance in conditions where the masker is not repeated is driven by factors related to overall skill with word recognition in noise, whereas an additional factor is at work in conditions where the masker is repeated.
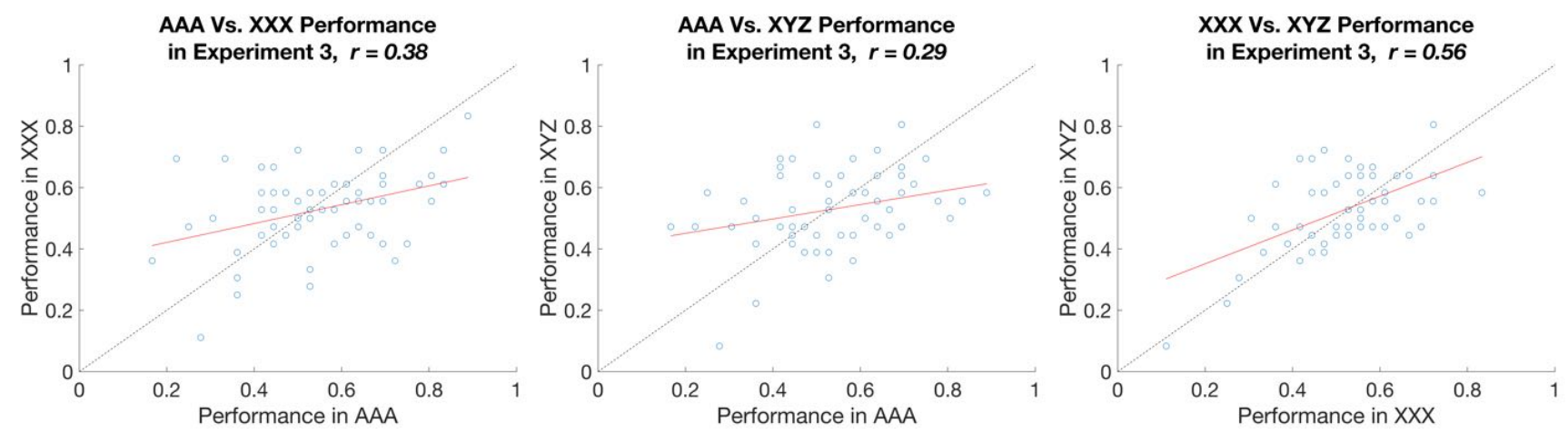

Figure 8: A weak correlation is observed between AAA and XYZ, while XXX exhibits a modest correlation with AAA. XXX is moderately correlated with XYZ. A least mean squares linear regression line is drawn in red and the identity line is drawn in black on all plots.

Fisher's z-transformation reveals XXX/XYZ to be a significantly stronger correlation than AAA/XYZ, $z=$ $1.75, p=.04$, but not significantly stronger than AAA/XXX, $z=1.22, p=.11$. We find no significant difference in correlation strength between AAA/XXX and AAA/XYZ, $z=.52, p=.30$.

Hierarchical regression reveals that XYZ again explains significant AAA variance, $F(1,58)=5.51, p<.05$, but XXX explains significantly more when included, $F(1,57)=4.60, p<.05$. XXX itself explains significant AAA variance, $F(1,58)=9.86, p<.005$, beyond which including XYZ does not explain significantly more, $F(1,57)=$ $.65, p=.42$. 


\subsubsection{Musical Experience Survey}

We use three variables measuring participants' musical experience in our analysis: years of formal training, hours per day spent listening to music, and current involvement in music. These respectively capture the amount of learned skills, degree of passive exposure, and condition of acquired skills.

We find no significant correlation between overall task performance and years of musical training, $r(58)=$ $.15, p=.12$, or hours per day spent listening to music, $r(58)=-.11, p=.80$. We also find no significant correlation in the difference between AAA and XYZ and years of formal training, $r(58)=-.05, p=.65$, nor average hours per day listening to music, $r(58)=-.15, p=.87$. Musical involvement was measured with a yes/no question and analyzed with a logistic classfier. The decision boundary maximizing accuracy simply classifies the data in one class, producing an accuracy of .77, its underlying class distribution, for both overall performance and AAA-XYZ difference, indicating no relationship with either.

\subsection{Discussion}

Repetition of the masker within a trial appears to have little impact on performance in the word recognition task, based on the lack of an effect on the means and the lack of any detectable effect on variances. Trials containing a stimulus repeated over the course of the experiment, in contrast, differed (although marginally) from both of the other conditions in the variance observed, and was only weakly to moderately correlated with the other conditions, suggesting that the repetition of the masker over the course of the experiment may be more important in driving individual differences. It is interesting to note that XXX explains significantly more AAA variance than baseline alone, despite their mutual lack of long-term information. While this hints at a possible effect of short-term information, the effect is not strong enough to drive significant findings in any of the other tests of central tendency or variance we perform in this paper (Experiments 1 and 5 mean effects notwithstanding). Lastly, musical experience did not correlate with overall word recognition performance nor with differences between the AAA condition and the XYZ baseline condition. Two conditions in this experiment (AAA and XYZ) were identical to Experiment 1, but did not produce the same difference in means, prompting us to conduct a direct replication of Experiment 1.

\section{Experiment 4}

Experiment 4 is an attempt at a direct replication of Experiment 1.

\subsection{Methods}

The experiment's design, stimuli, and procedure are identical to Experiment 1, except the substitution of a musical experience survey for the tests of working memory and inhibitory control. Sixty new subjects were recruited from the same population and in the same manner as previous experiments.

\subsection{Results}

The same mixed-effects logistic regression model from Experiment 1 (see $\$ 2.2 .1$ for details) reveals no significant mean difference between AAA and XYA, $\beta=-.01, z=-0.21, p=.84$, and only a marginally significant difference between AAA and XYZ, $\beta=-.12, z=-1.79, p=.07$. No significant difference was observed between XYA and $\mathrm{XYZ}, \beta=-.11, z=-1.58, p=.11$.

Variance Differences Bartlett's test reveals the variance of AAA, $s=.17$, to be significantly greater than that of XYZ, $s=.12, T(1)=6.61, p=.01$, and the variance of XYA, $s=.16$, to be significantly greater than XYZ as well, $T(1)=4.33, p=.04$, as shown in Figure 9. The variances of AAA and XYA do not significantly differ, $T(1)=.25, p=.62$. 

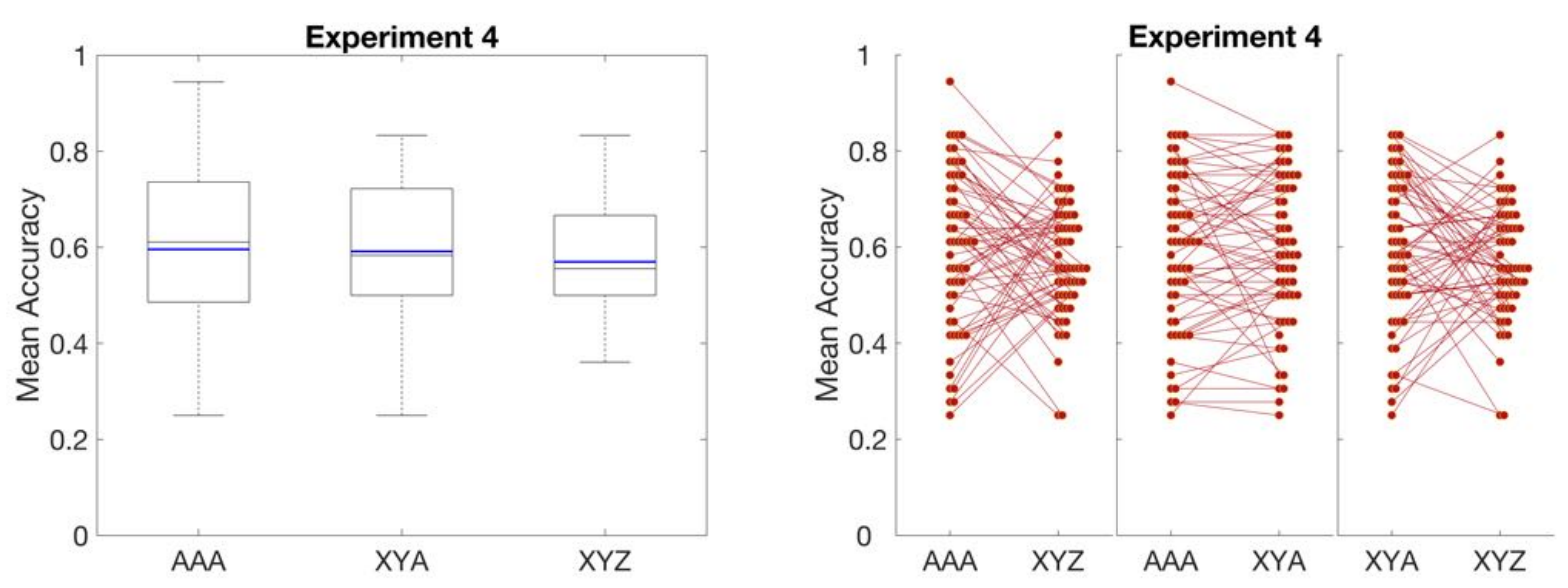

Figure 9: Neither previously significant difference between condition means (blue, left) replicates. The short-term and long-term condition, AAA, does not differ significantly from the long-term only condition, XYA, and differs only marginally from baseline, XYZ. The lack of significant difference between the latter two conditions replicates from Experiment 1. The distribution variances of AAA and XYA both differ significantly from XYZ but not each other, replicating the findings of Experiment 1 and 2. Lines (right) again connect participant performances and represent correlation between conditions.

Condition performance correlations As in Experiment 1, AAA performance is a strong predictor of XYA performance, $r(58)=.7, p<.0001$, (Fig. 10). No significant correlations are observed between XYZ and AAA, $r(58)=.11, p=.20$, and between XYZ and XYA, $r(58)=.07, p=.29$.
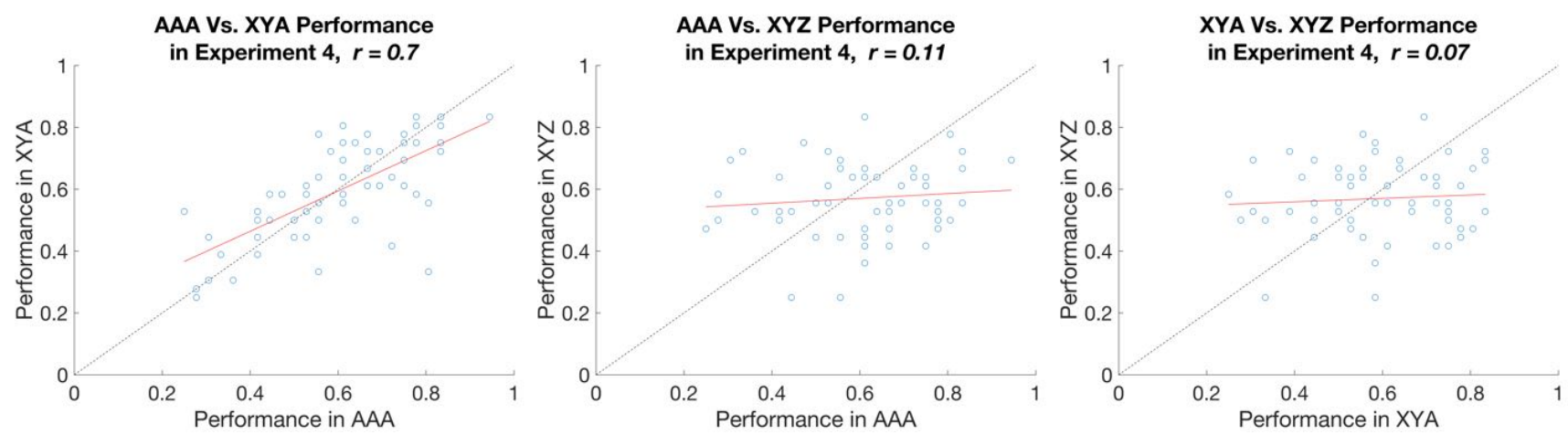

Figure 10: Experiment 4 replicates the strong correlation between conditions with long-term information (AAA and XYA). The finding that both AAA and XYA are poor predictors of baseline performance (XYZ) also replicates. A least mean squares linear regression line is drawn in red and the identity line is drawn in black on the plots for reference.

We replicate the correlation findings of Experiment 1 and again find AAA/XYA to be a significantly stronger correlation than AAA $/ \mathrm{XYZ}, z=4.00, p<.0001$, as well as XYA/XYZ, $z=4.20, p<.0001$. We do not find a significant difference in the strength of correlations between AAA/XYZ and XYA/XYZ, $z=.20, p=.42$.

We replicate the hierarchical regression of Experiment 1 and again find XYA to explain significant variance in AAA performance, $F(1,58)=54.55, p<.0001$, and significantly more variance than XYZ alone, $F(1,57)=$ $53.02, p<.0001$. Here, XYZ again does not explain significantly more variance than XYA, $F(1,57)=.38, p=.54$, and moreover fails to explain a significant amount of variance alone, $F(1,58)=.70, p=.41$.

\section{Musical Experience}

As in Experiment 3, amount of musical training is a poor predictor of AAA benefit, $r(58)=.17, p=.10$. Additionally, average daily listening time is again a poor predictor of AAA benefit, $r=-.03, p=.60$ as well. Current musical involvement is also uninformative as a predictor of AAA benefit, once again returning all the data as one class and achieving the underlying class distribution, .7, as its accuracy. 


\subsection{Discussion}

When considering differences in measures of central tendency, a very different pattern was observed here than in Experiment 1, where the condition in which maskers were repeated within the trial (AAA) differed from both a condition that comprised all novel maskers (XYZ), and a condition in which the masker was repeated over the course of the experiment (XYA). In Experiment 4, performance did not differ significantly between AAA and XYA, and neither differed significantly from XYZ, although they did differ numerically. This failure to replicate prompted the exploratory analyses reported throughout the paper, i.e., comparisons of the variance across conditions, and the correlation among conditions. These analyses produce similar results in Experiment 1 and the replication: conditions in which the masker is repeated throughout the experiment (AAA and XYA) produce greater variability between participants, and performance in these conditions is strongly correlated, whereas performance in the condition in which the masker is not repeated is only weakly correlated with both. This is supported throughout by hierarchical regression analyses, which are most strongly seen here: baseline performance does not explain significant variance in AAA performance but XYA both explains significant variance and significantly more than baseline.

\section{$6 \quad$ Experiment 5}

Thus far, the results clearly demonstrate that repeating the masking pattern at both the single trial level, and over the course of the experiment increases the variance by participants on trials including the repeated pattern. Although performance on trials with repeated maskers was numerically more accurate in each experiment, this effect was only significant in Experiment 1, and was not reproduced in a direct replication. In order to create a firmer foundation for future investigations, we sought to increase the strength of the effect by increasing the number of repetitions for repeated masking patterns from three to five. We reasoned that this would be predicted to increase the advantage for trials with the repeated masker, whether this arises from repetition within trial or across trials.

\subsection{Methods}

\section{Participants and Procedure}

The participants and procedure are the same as in previous experiments with 60 participants in this study.

\subsubsection{Stimuli}

The stimuli are created in the same manner as in experiment 1, with the exception that we present 4 masking patterns prior to target presentation giving us 5 masking patterns in all. Given the fivefold repetition, the repeating condition is now condition AAAAA, the glimpsing control condition is now condition VWXYA, and the baseline condition is now condition VWXYZ.

\subsection{Results}

\subsubsection{Hypothesis Testing}

The same multilevel mixed-effects logistic regression model as in previous experiments reveals a significant main effect of condition, $\chi^{2}(2, N=60)=17.63, p=.0001$. Pairwise comparison between conditions shows a marginally significant difference between AAAAA and VWXYA, $\beta=-.11, z=-1.63, p=.10$. The model also reveals significant differences between AAAAA and VWXYZ, $\beta=-.29, z=-4.16, p<.001$, and VWXYA and VWXYZ, $\beta=-.17, z=$ $-2.53, p=.01$. 

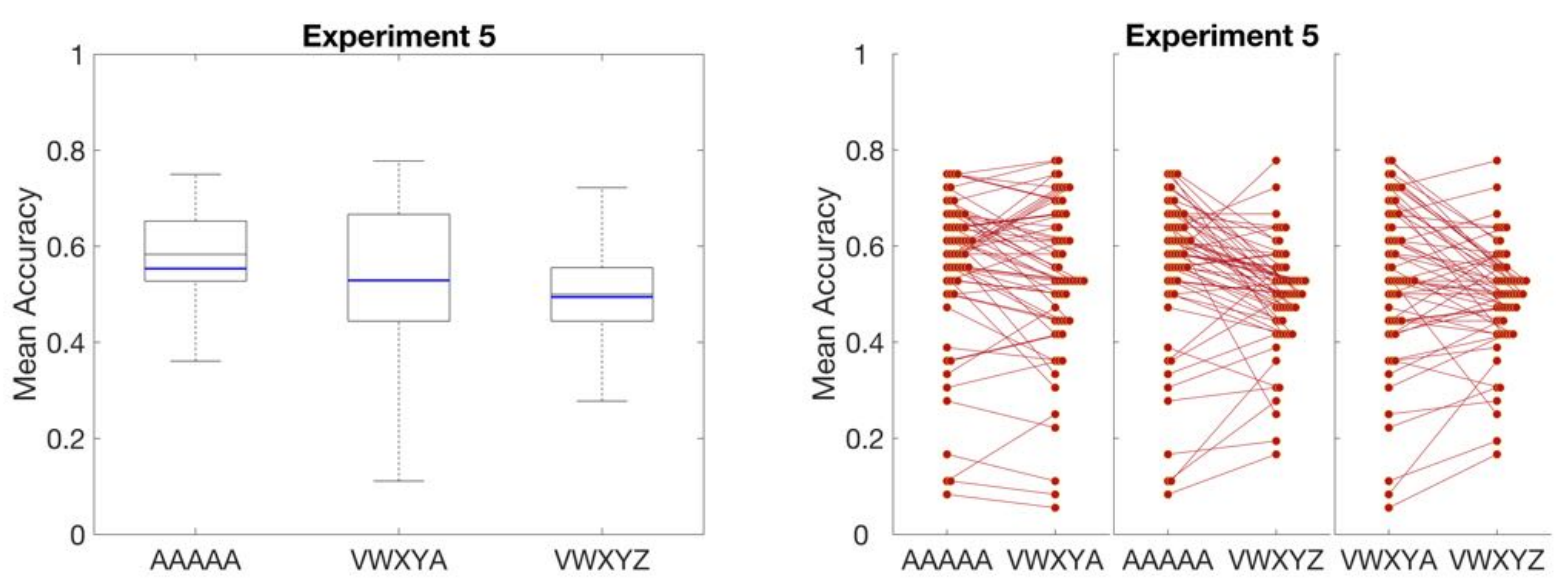

Figure 11: We observe significant mean differences (blue, left) between the baseline condition and both conditions containing pattern A. We also observe a marginally significant difference between the repeated and recurring condition over just the recurring condition. Additionally, we observe significant variance differences (see vertical distributions, right) between the conditions containing pattern A and the baseline condition.

\subsubsection{Exploratory Analyses}

Variance Differences We again test for unequal variances using Bartlett's, which reveals a significant variance difference between AAAAA $(s=.159)$ and $\operatorname{VWXYZ~}(s=.115), T(1)=5.98, p=.01$, a small effect size, $d=.43$ (Cohen, 1977). We also observe a significant variance difference between VWXYA $(s=.168)$ and VWXYZ, $T(1)=$ $8.05, p<.005$, a small effect size, $d=.24$. We find no significant difference between the variances of AAAAA and VWXYA.

Condition performance correlations As before, we test for correlations between conditions. We find significant strong correlations among all conditions (see Figure 12 for individual correlations). We also test for differences in correlations strengths and find a significant difference between the conditions with pattern $\mathrm{A}$ as the masker, AAAAA/VWXYA $(r=.83)$, and between the repeated condition and baseline, AAAAA/VWXYZ $(r=.64), z=$ $2.31, p=.01$. We also find a marginally significant difference between the conditions with pattern $\mathrm{A}$ as the masker and between the recurring condition and baseline, VWXYA/VWXYZ $(r=.71), z=1.6, p=.055$. We find no significant difference between correlation strengths of either pattern A condition with baseline (i.e. AAAAA/VWXYZ Vs. VWXYA/VWXYZ), $z=-0.71, p=0.76$.
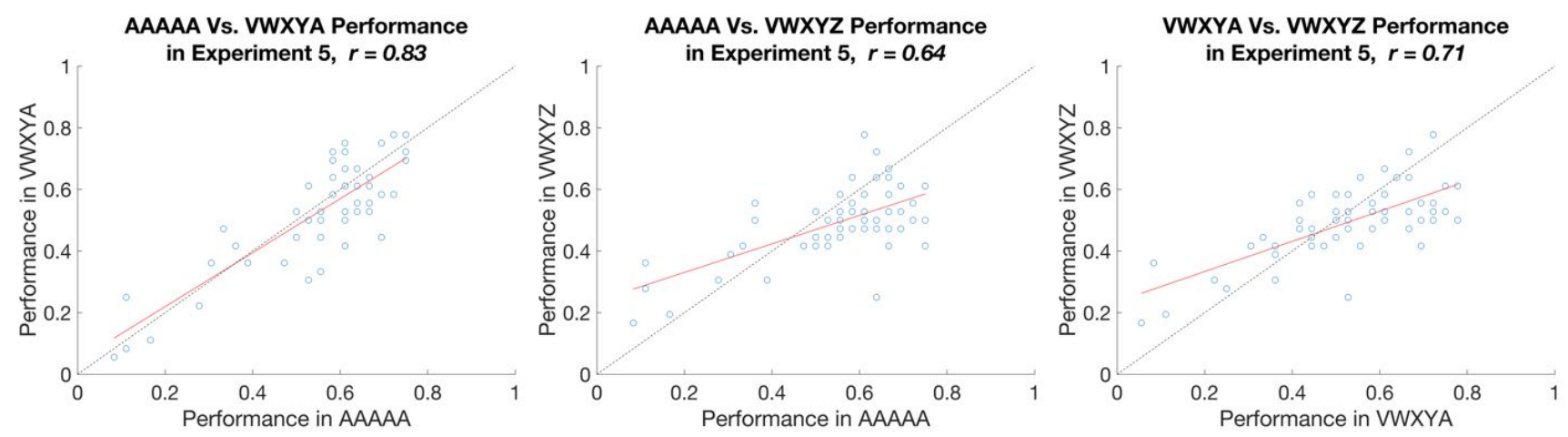

Figure 12: We observe a very strong correlation between conditions with pattern A as a masker. The correlations observed here are stronger than others in this paper. The strength of the correlation between pattern A conditions is significantly stronger than between either and baseline. A red LMS line and black identity line are included for reference. 


\subsection{Discussion}

Increasing the number of repetitions for the repeated masking pattern produces large, significant differences between conditions in which the repeated masker is used (both AAAAA and VWXYA) and the baseline condition with random maskers throughout. The generality of the effect in conditions including the repeated masker, along with the increased individual variability in both conditions and the strong correlation between them suggests that performance in these conditions is in part attributable to the same mechanism. This in turn suggests a strong role for "long-term" processes involving the learning of the "A" pattern over the course of the experiment. We find weaker evidence for the involvement of "short-term" processes engaged over the course of an individual trial: performance in trials where the masker was presented repeatedly differed only marginally from trials on which the repeated masker was presented only once.

\section{General Discussion}

Our initial hypothesis was that participants would be able to use repeating temporal information within a trial (short-term information) to overcome masking and improve their performance in a word recognition in noise task. Although initial analyses of measures of central tendency supported this hypothesis in Experiment 1, the effect is subtle and does not always achieve statistical significance. As seen in Table 1, the advantage for the AAA condition over other conditions observed in Experiment 1 is not observed consistently across experiments. A final experiment, in which the masking pattern was repeated more frequently, did produce a statistically significant effect, as in Experiment 1. Interestingly, even in cases where significant differences in mean performance were not observed, the use of repeating maskers increased variability, and performance across conditions including repeated maskers was moderately to strongly correlated, suggesting that some as yet unidentified individual difference in skill, capacity, or strategy is exaggerated by the repetition of the masking pattern, as shown in Tables 3 and 4, respectively. Specifically, conditions in which the masker was repeated throughout the experiment (AAA and XYA) were consistently found to increase variance when compared with conditions in which the stimulus was not repeated (XYZ), or was repeated only within a trial (XXX). Further, in all four experiments in which both conditions were included, strong correlations were observed between the AAA and XYA conditions. This is supported by hierarchical regression which repeatedly finds XYA to explain significantly more variance in AAA performance than baseline alone.

On balance, our findings suggest that the availability of recurring information across trials (long-term information), rather than repeating information within trials (short-term information), is important in determining performance in each condition. Direct tests designed to evaluate the contribution of streaming within a trial, as when silences were inserted between maskers in the preamble in Experiment 2, or the repetition of the masker within a trial, as in Experiment 3, did not produce evidence for the use of short-term information. Further, we note that in Experiment 3, the "A" masker was repeated less frequently than in the other experiments, and in that experiment we observed the smallest effect sizes for comparisons between the AAA condition and others, in terms of both differences in the mean and the variance, as shown in Tables 2 and 3. Experiment 3 presents another noteworthy finding - the correlation between conditions that lack long-term information (XXX and XYZ) is smaller than between those that contain it (AAA and XYA, see Table 4). Thus, it may be that the factors driving overall performance in the task are weaker determinants of individual differences than the additional factor involved when maskers are repeated. It is also possible that the weaker correlation is simply a statistical artifact of the lower variability in conditions without repeated maskers (Nikolić et al., 2012; Alexander et al., 1984; Aguinis and Whitehead, 1997), but this begs the question of why the variances are greater specifically in conditions with repeated maskers.

Performance Means by Condition
\begin{tabular}{|l|c|c|c|c|}
\hline & AAA & XYA & XXX & XYZ \\
\hline Experiment 1 & .66 & .61 & - & .61 \\
\hline Experiment 2 & .58 & .58 & - & .56 \\
\hline Experiment 3 & .55 & - & .53 & .53 \\
\hline Experiment 4 & .60 & .59 & - & .57 \\
\hline Experiment 5 & .55 & .53 & - & .49 \\
\hline
\end{tabular}

Table 1: Mean performance by condition appears fairly variable and unreliable across experiments. Empty cells indicate the condition did not occur in that experiment. 
Cohen's d Across Experiments

\begin{tabular}{|c|c|c|c|}
\hline & Exp & AAA & XYZ \\
\hline \multirow{3}{*}{ XYA } & 1 & .26 & .03 \\
\cline { 2 - 4 } & 2 & -.04 & .17 \\
\cline { 2 - 4 } & 4 & .03 & .16 \\
\cline { 2 - 4 } & 5 & .15 & .24 \\
\hline XXX & 3 & .14 & -.03 \\
\hline \multirow{4}{*}{ XYZ } & 1 & .31 & - \\
\cline { 2 - 3 } & 2 & .12 & - \\
\cline { 2 - 3 } & 3 & .11 & - \\
\cline { 2 - 3 } & 4 & .18 & - \\
\cline { 2 - 3 } & 5 & .43 & - \\
\hline
\end{tabular}

Table 2: Cohen's $d$ (Cohen, 1988) reveals that normalized mean differences also vary greatly across experiments. The XYA vs. XXX comparison is absent as the two were mutually exclusive. Together with Table 1, these results indicate that central tendency is not a robust indicator in our experiments.

Standard Deviations by Condition

\begin{tabular}{|l|c|c|c|c|}
\hline & AAA & XYA & XXX & XYZ \\
\hline Experiment 1 & .18 & .17 & - & .14 \\
\hline Experiment 2 & .16 & .16 & - & .11 \\
\hline Experiment 3 & .16 & - & .13 & .13 \\
\hline Experiment 4 & .17 & .16 & - & .12 \\
\hline Experiment 5 & .16 & .17 & - & .12 \\
\hline
\end{tabular}

Table 3: Standard deviation by condition is relatively stable, in contrast with condition means. These results, and their levels of significance, provide evidence that variance is a robust metric, grouping conditions along the basis of long-term information: AAA with XYA and XXX with XYZ. Empty cells indicate the condition did not occur in that experiment.

Condition Correlations
\begin{tabular}{|c|c|c|c|}
\hline & Exp & AAA & XYZ \\
\hline \multirow{4}{*}{ XYA } & 1 & .79 & .26 \\
\cline { 2 - 4 } & 2 & .79 & .53 \\
\cline { 2 - 4 } & 4 & .70 & .07 \\
\cline { 2 - 4 } & 5 & .83 & .71 \\
\hline XXX & 3 & .38 & .56 \\
\hline \multirow{4}{*}{ XYZ } & 1 & .32 & - \\
\cline { 2 - 4 } & 2 & .51 & - \\
\cline { 2 - 3 } & 3 & .29 & - \\
\cline { 2 - 3 } & 4 & .11 & - \\
\cline { 2 - 3 } & 5 & .64 & - \\
\hline
\end{tabular}

Table 4: Condition correlations are reliable across experiments in their strength (AAA \& XYA) or weakness (AAA \& XYZ and XYA \& $\mathrm{XYZ)}$. These robust findings, as well as Experiment 3's weak correlation of XXX with AAA and moderate correlation with XYZ, support the long-term information account provided by variance (see Table 3). Experiment 2's higher correlations for AAA \& XYZ and XYA \& XYZ may be due to the silences between patterns, making it not directly comparable to other experiments. The XYA \& XXX correlation is absent as the two conditions were mutually exclusive.

We collected ancillary data about people's working memory and executive function abilities (Experiment 1) and about their musical training and experience (Experiments 3 and 4), but found no evidence for correlations of these measures with the size or direction of the effect of repeated information in the masker on word recognition. One goal of future work with these phenomena will be to identify other sources of variability that contribute to the increased variance in performance when maskers are repeated throughout an experiment. For example, long-term information necessitates a long-term memory representation of the object, given the time-course of the experiment and the inability to rehearse noise to keep it active in working memory (Demany et al., 2001; Kaernbach, 2004). Furthermore, this memory storage must represent pattern A alone so that the speech with which it co-occurs in previous trials does not interfere with its application in further trials, analogous to the separate storage of each voice in polyphonic music (Fujioka et al. 2005, for review see Trainor et al. 2014). Given the many dimensions along which our maskers differ from the target speech (timbre, envelope, spectral constancy, etc.), it is quite possible that they 
are treated as a separate "voice" - or at least a distinct object - for the purposes of memory storage.

Because of the increased variability in the AAA and XYA conditions, and the correlations between them across experiments, we hypothesize that the increased variance may be due to differential effects of repeating the masking pattern on two distinct subsets of participants. Individuals may differ in their ability to form distinct memory representations for simultaneously presented objects (words and masking patterns), so that for some participants the memory of the masker improves performance, whereas for others, memory of the prior presentation of the masker produces proactive interference.

A possible mechanism for this differential account comes from load theory (Lavie 1995, 2000, for review see Lavie 2010, 2005): when cognitive load remains below a person's capacity, they process all information automatically and non-selectively. It is only when load exceeds capacity that selection occurs and processing is narrowed to the object of focus. As it relates to our task, participants whose cognitive capacities are not exhausted by task demands automatically process all information in the stimuli - both the speech and the noise. However, participants whose capacities are exceeded by task demands will necessarily constrain attention to only the target task of speech perception - disregarding the information contained in the noise. In this account, below-capacity participants can devote resources to capitalizing on the information present in AAA and XYA, while above-capacity participants are likely to be adversely affected by additional surplus information they are unable to make use of.

Given the load theory account presented above, this individual variability is likely to occur along the lines of cognitive capacity. It is thus surprising that the working memory and executive function measures we obtained were not predictive of long-term information benefit. This is likely explained by the visual modality of the ancillary tasks, which differs from the auditory modality in capacity and processing (Cohen et al., 2009). Additionally, Cohen et al. (2011) found that musicians' superior auditory memory did not extend to the visual modality, implying that we did not measure the dimension of interest for an auditory task like ours. Our results support Stenbäck et al. (2015)'s findings that similar measures of executive function and working memory were uncorrelated with each other and parallel conditions in their word recognition task (cf. Rönnberg et al. (2010); Sörqvist (2010)). Given musicians' superior auditory memory (Cohen et al., 2011), musical experience also represented a good candidate for an informative covariate. However, our measurements of music education, involvement, and consumption all proved to be uninformative with regards to task performance. This may be explained in part by Honing et al. (2009)'s findings that beat induction and rhythm tracking mechanisms are at least partially innate, and are not in fact preferentially present in musicians over non-musicians (cf. Thompson et al. 2015). While we measured musicianship as a proxy for beat tracking ability, we plan in future studies to directly assess this ability and correlate it with task performance. This is motivated by findings that both beat tracking and beat production ability moderate speech perception in noise performance (Slater and Kraus 2016; Slater et al. 2018), possibly by mitigating the effect of backward masking (Tierney and Kraus, 2013).

\section{References}

Aguinis, H. and Whitehead, R. (1997). Sampling variance in the correlation coefficient under indirect range restriction: Implications for validity generalization. Journal of Applied Psychology, 82(4):528-538.

Akeroyd, M. A. (2008). Are individual differences in speech reception related to individual differences in cognitive ability? A survey of twenty experimental studies with normal and hearing-impaired adults. International Journal of Audiology, 47(sup2):S53-S71.

Alexander, R. A., Carson, K. P., Alliger, G. M., and Barrett, G. V. (1984). Correction for Restriction of Range when Both X and Y are Truncated. Applied Psychological Measurement, 8(2):231-241.

Andreou, L.-V., Kashino, M., and Chait, M. (2011). The role of temporal regularity in auditory segregation. Hearing Research, 280(1-2):228-235.

Bartlett, M. S. (1937). Properties of Sufficiency and Statistical Tests. Proceedings of the Royal Society of London. Series A, Mathematical and Physical Sciences, 160(901):268-282.

Boersma, P. and Weenink, D. (2012). Praat: doing phonetics by computer. 
Bregman, A. S. (1990). Auditory scene analysis: the perceptual organization of sound. MIT Press, Cambridge, Mass.

Brungart, D. S. (2001). Informational and energetic masking effects in the perception of two simultaneous talkers. The Journal of the Acoustical Society of America, 109(3):1101-1109.

Clopper, C. G. and Pisoni, D. B. (2006). The Nationwide Speech Project: A new corpus of American English dialects. Speech Communication, 48:633-644.

Cohen, J. (1977). Statistical Power Analysis for the Behavioral Sciences. Elsevier, Hoboken.

Cohen, J. (1988). Statistical Power Analysis for the Behavioral Sciences. Routledge. Google-Books-ID: 2v9zDAsLvA0C.

Cohen, M. A., Evans, K. K., Horowitz, T. S., and Wolfe, J. M. (2011). Auditory and visual memory in musicians and nonmusicians. Psychonomic bulletin 83 review, 18(3):586-591.

Cohen, M. A., Horowitz, T. S., and Wolfe, J. M. (2009). Auditory recognition memory is inferior to visual recognition memory. Proceedings of the National Academy of Sciences of the United States of America, 106(14):6008-6010.

Cooke, M. (2003). Glimpsing speech. Journal of Phonetics, 31(34):579-584.

Cusack, R., Decks, J., Aikman, G., and Carlyon, R. P. (2004). Effects of Location, Frequency Region, and Time Course of Selective Attention on Auditory Scene Analysis. Journal of Experimental Psychology: Human Perception and Performance, 30(4):643-656.

Demany, L., Clément, S., and Semal, C. (2001). Does auditory memory depend on attention? In D.J. Breebaart, A.J.M. Houtsma, A. Kohlrausch, V.F. Prijs, and R. Schoonhoven, editors, Physiological and Psychophysical Bases of Auditory Function, pages 461-467. Shaker Publishing BV, Maastricht (The Netherlands).

Dryden, A., Allen, H. A., Henshaw, H., and Heinrich, A. (2017). The Association Between Cognitive Performance and Speech-in-Noise Perception for Adult Listeners: A Systematic Literature Review and Meta-Analysis. Trends in Hearing.

Festen, J. M. and Plomp, R. (1990). Effects of fluctuating noise and interfering speech on the speechreception threshold for impaired and normal hearing. The Journal of the Acoustical Society of America, 88(4):1725-1736.

Fisher, R. A. (1915). Frequency distribution of the values of the correlation coefficient in samples from an indefinitely large population. Biometrika, 10(4):507-521.

Fujioka, T., Trainor, L. J., Ross, B., Kakigi, R., and Pantev, C. (2005). Automatic Encoding of Polyphonic Melodies in Musicians and Nonmusicians. Journal of Cognitive Neuroscience, 17:1578-1592.

Hollingworth, A. (2005). The Relationship Between Online Visual Representation of a Scene and Long-Term Scene Memory. Journal of Experimental Psychology: Learning, Memory, and Cognition, 31(3):396-411.

Honing, H. (2012). Without it no music: beat induction as a fundamental musical trait. Annals of the New York Academy of Sciences, 1252(1):85-91.

Honing, H., Ladinig, O., Háden, G. P., and Winkler, I. (2009). Is Beat Induction Innate or Learned?: Probing Emergent Meter Perception in Adults and Newborns using Event-related Brain Potentials. Annals of the New York Academy of Sciences, 1169(1):93-96.

Kaernbach, C. (2004). The Memory of Noise. Experimental Psychology, 51(4):240-248.

Kidd, G., Mason, C. R., Richards, V. M., Gallun, F. J., and Durlach, N. I. (2008). Informational Masking. In Yost, W. A., Popper, A. N., and Fay, R. R., editors, Auditory Perception of Sound Sources, Springer Handbook of Auditory Research, pages 143-189. Springer US, Boston, MA.

Lander-Portnoy, M. (2016). Release from energetic masking caused by repeated patterns of glimpsing windows. In Interspeech 2016, pages 1672-1676.

Lavie, N. (1995). Perceptual load as a necessary condition for selective attention. Journal of Experimental Psychology: Human Perception and Performance, 21(3):451-468. 
Lavie, N. (2000). Selective attention and cognitive control: Dissociating attentional functions through different types of load. In Monsell, S. and Driver, J., editors, In: Monsell, S and Driver, J, (eds.) UNSPECIFIED (175 - 194). M I T PRESS (2000), pages 175-194. M I T PRESS.

Lavie, N. (2005). Distracted and confused?: Selective attention under load. Trends in Cognitive Sciences, 9(2):75-82.

Lavie, N. (2010). Attention, Distraction, and Cognitive Control Under Load. Current Directions in Psychological Science, 19(3):143-148.

Lowry, R. (2001). Two Correlation Coefficients: http://vassarstats.net/rdiff.html.

Mattys, S. L., Davis, M. H., Bradlow, A. R., and Scott, S. K. (2012). Speech recognition in adverse conditions: A review. Language and Cognitive Processes, 27(7-8):953-978.

Miller, G. A. and Licklider, J. C. R. (1950). The Intelligibility of Interrupted Speech. The Journal of the Acoustical Society of America, 22(2):167-173.

Nikolić, D., Mureşan, R. C., Feng, W., and Singer, W. (2012). Scaled correlation analysis: a better way to compute a cross-correlogram. European Journal of Neuroscience, 35(5):742-762.

Pollack, I. (1975). Auditory informational masking. The Journal of the Acoustical Society of America, 57(S1):S5-S5.

Rönnberg, J., Rudner, M., Lunner, T., and Zekveld, A. (2010). When cognition kicks in: Working memory and speech understanding in noise. Noise and Health, 12(49):263-269.

Scott, S. K., Rosen, S., Wickham, L., and Wise, R. J. S. (2004). A positron emission tomography study of the neural basis of informational and energetic masking effects in speech perception. The Journal of the Acoustical Society of America, 115(2):813-821.

Shamma, S. A., Elhilali, M., and Micheyl, C. (2011). Temporal coherence and attention in auditory scene analysis. Trends in Neurosciences, 34(3):114-123.

Sheft, S. and Yost, W. A. (2008). Method-of-adjustment measures of informational masking between auditory streams. The Journal of the Acoustical Society of America, 124(1):EL1-EL7.

Slater, J. and Kraus, N. (2016). The role of rhythm in perceiving speech in noise: a comparison of percussionists, vocalists and non-musicians. Cognitive Processing, 17(1):79-87.

Slater, J., Kraus, N., Carr, K. W., Tierney, A., Azem, A., and Ashley, R. (2018). Speech-in-noise perception is linked to rhythm production skills in adult percussionists and non-musicians. Language, Cognition and Neuroscience, 33(6):710-717.

Smiarowski, R. A. and Carhart, R. (1975). Relations among temporal resolution, forward masking, and simultaneous masking. The Journal of the Acoustical Society of America, 57(5):1169-1174.

Snyder, J. S., Alain, C., and Picton, T. W. (2006). Effects of Attention on Neuroelectric Correlates of Auditory Stream Segregation. Journal of Cognitive Neuroscience, 18(1):1-13.

Sörqvist, P. (2010). The role of working memory capacity in auditory distraction: A review. Noise and Health, 12(49):217-224.

Stenbäck, V., Hällgren, M., Lyxell, B., and Larsby, B. (2015). The Swedish Hayling task, and its relation to working memory, verbal ability, and speech-recognition-in-noise. Scandinavian Journal of Psychology, 56(3):264-272.

Sun, H.-M. and Gordon, R. D. (2010). The influence of location and visual features on visual object memory. Memory E Cognition, 38(8):1049-1057.

Thompson, E. C., White-Schwoch, T., Tierney, A., and Kraus, N. (2015). Beat Synchronization across the Lifespan: Intersection of Development and Musical Experience. PLOS ONE, 10(6):e0128839.

Tierney, A. T. and Kraus, N. (2013). The ability to tap to a beat relates to cognitive, linguistic, and perceptual skills. Brain and Language, 124(3):225-231. 
Trainor, L. J., Marie, C., Bruce, I. C., and Bidelman, G. M. (2014). Explaining the high voice superiority effect in polyphonic music: Evidence from cortical evoked potentials and peripheral auditory models. Hearing Research, 308:60-70.

Treisman, A. M. (1964). Selective Attention In Man. British Medical Bulletin, 20(1):12-16.

Widin, G. P. and Viemeister, N. F. (1979). Intensive and temporal effects in puretone forward masking. The Journal of the Acoustical Society of America, 66(2):388-395.

Winkler, I., Haden, G. P., Ladinig, O., Sziller, I., and Honing, H. (2009). Newborn infants detect the beat in music. Proceedings of the National Academy of Sciences, 106(7):2468-2471. 